\title{
LOS ESTUDIANTES UNIVERSITARIOS ANTE LAS REDES SOCIALES: CUESTIONES DE USO Y AGRUPACIÓN EN ESTRUCTURAS ELITISTAS O PLURALISTAS
}

\section{UNIVERSITY STUDENTS TO THE SOCIAL NETWORKS: ISSUES AND USER GROUP IN ELITIST STRUCTURE OR PLURAL}

\author{
AUTORES \\ Alejandro Tapia Frade: Profesor Doctor Adjunto, Universidad Europea Miguel de Cervantes. Madrid \\ (España) \\ atapia@uemc.es
}

Begoña Gómez Nieto: Profesora Doctora Adjunta, Universidad Europea Miguel de Cervantes. Madrid (España)

mbgomez@uemc.es

Jose María Herranz de la Casa: Profesor Doctor Adjunto y Director de Comunicación, Universidad Europea Miguel de Cervantes. Madrid (España)

jmherranz@uemc.es

Mónica Matellanes Lazo: Profesora Doctora Adjunta, Universidad Europea Miguel de Cervantes. Madrid (España)

mmatellanes@uemc.es

\section{RESUMEN}

El desarrollo de las redes sociales facilita la comunicación de diferentes usuarios de un modo globalizado y muchas veces asíncrono, en el que comparten y transmiten opiniones de diversa índole. Este trabajo se centra, dentro de este complejo fenómeno, específicamente en el uso que una parte de los nativos digitales (Fumero y Hervás, 2008:65) hacen de Facebook, MySpace, Tuenti, Hi5, Ringo, Bebo y un amplio número de redes sociales.

Profundizando más en el objeto de estudio, tratamos de determinar la estructura de las redes sociales virtuales en el caso de comunidades creadas a partir de estas aplicaciones, recuperando la idea esencial de estructura de comunidades divulgada en los años $50 \mathrm{y}$ 60 entre otros por Dahl y Hunter, que dividían las estructuras en dos tipos puros: elitistas y pluralistas. Tratamos pues de determinar la existencia de líderes dentro de 
estas estructuras virtuales, esenciales para la delimitación de estas estructuras, y examinamos el carácter multiespacial y asíncrono de estas relaciones.

Este artículo presenta los resultados de un estudio empírico realizado entre estudiantes de la Universidad Europea Miguel de Cervantes de Valladolid y de la Universidad Francisco de Vitoria de Madrid, donde se analizan los planteamientos expuestos anteriormente.

\section{PALABRAS CLAVE}

Internet - Redes sociales - Estructura interna - Pluralismo - Elitismo

\section{ABSTRACT}

The development of the social networks facilitate the communication of different users in a globalized world, which transmit and share a wide variety of opinions. This work is within this complex phenomenon, specifically on the use that part of the digital natives (Fumero y Hervás, 2008:65) do with Facebook, MySpace, Tuenti, Hi5, Ringo, Bebo and a large number of social networks.

Deeper into the subject of study, we try to determine the structure of the community in case of virtual communities created from these applications, recovering the key structure of communities published in the 50s and 60s, among others, by Hunter and Dahl, which split into two structures cigars: elitist and pluralist. Therefore, determine the existence of leaders within these virtual structures and examine the nature and of these relationships.

This article presents the results of an empirical study conducted among students at the Universidad Europea Miguel de Cervantes de Valladolid and the University Francisco de Vitoria in Madrid, where some of the approaches discussed above.

\section{KEY WORDS}

Internet - Social networks - Internal structure - Pluralism - Elitism.

\section{ÍNDICE}
$\boldsymbol{2}$ 1. INTRODUCCIÓN
2. 2. METODOLOGÍA
$\boldsymbol{\sim}$ 3. RESULTADOS 
3.1 Las Redes Sociales Usadas

3.2 Nomadismo Virtual

3.3 Estructura en redes sociales

\section{$\boldsymbol{\sim}$ 4. CONCLUSIONES Y DISCUSIÓN DE RESULTADOS \\ 2 5. BIBLIOGRAFÍA}

\section{INTRODUCCIÓN}

En la última década la revolución y notable desarrollo de Internet ha influenciado en los procesos de comunicación y generación e intercambio de información a través de la red. La evolución de la nueva Web 2.0 es evidente y son cada vez más los usuarios que reafirman su uso en las nuevas redes sociales, especialmente intenso en el caso de los españoles, aunque con un escaso grado de tecnificación (Eroski Consumer, 2010). Esta nueva perspectiva nos acerca a diferentes paradigmas comunicacionales que determinan la interacción entre diversos usuarios que navegan por la red.

La facilidad de acceso y de uso en Internet se destaca en los últimos años, lo que facilita el crecimiento de uso de las nuevas redes sociales. Un estudio realizado por Zed Digital (2008), señala que el 50\% de los internautas son usuarios habituales de dichas redes. Según el informe, dos de cada tres reconocen que las utilizan para realizar recomendaciones sobre marcas.

En este contexto se consolida la conocida Web 2.0, siendo las redes sociales, el máximo exponente de un fenómeno que ha trascendido a los primeros usos informáticos en los años noventa. Las redes sociales son un fenómeno flamante y clave para entender los próximos modelos de comunicación interpersonal.

Exitosas redes sociales como, Facebook, MySpace o tuenti potencian la conexión y relación de multitud de grupos que pueden compartir perfiles muy parecidos. MySpace es el líder a nivel mundial, mientras que Facebook ocupa el segundo puesto (Sánchez Revilla, 2008). En España la red líder es Facebook, seguida de tuenti quien lidera el número uno de internautas (IAB, 2009).

Facebook destaca como buscador de gente y transmite seriedad, originalidad y presencia en medios de comunicación. Por otro lado, tuenti aparece con fuerza entre los jóvenes de 18-24 años. Destaca su carácter nacional, "privado" y "exclusivo", su carácter poco mediático, lúdico y adolescente. Es necesario que alguien te invite para poder ingresar. Por tanto, los contactos suelen ser gente cercana y/ o conocida (Zed Digital, 2008).

Es indudable el protagonismo que está dejando la impronta llegada de estas plataformas virtuales entre la sociedad. Las posibilidades que ofrecen son muchas y 
algunas de ellas utilizan estructuras y criterios más complejos; la gran mayoría son utilizadas como mero entretenimiento, diversión y contacto con amigos y conocidos, mientras que otras están dirigidas a otros públicos con finalidades y funciones de tipo más profesional y laboral (como la red social Linkedin, pensada para un público más adulto).

Las redes sociales también pueden aportar información de cómo funcionan las estructuras de liderazgo entre los diferentes grupos y subgrupos que se comunican en estos espacios virtuales. El análisis de dichas estructuras puede facilitar el estudio de diferentes perfiles y actitudes de individuos en las diversas conexiones que se dan en Internet. En general, aunque principalmente para los targets más jóvenes (entre 18 y 24 años), destaca cómo este entramado de redes, más allá de sus funcionalidades, está aportando valores como:

-Autoafirmación; desde las diferencias y similitudes con los demás.

-Reconocimiento social; la "popularidad" medida en función de los contactos que se tengan.

-Inclusión Social; sentirse parte de uno o varios grupos determinados.

En la actualidad, gracias al conocimiento de cómo funcionan las redes se explican muchas de las conductas y actitudes humanas que tienen lugar en espacios de interacción física. Los públicos más adolescentes y jóvenes son los que se ven más influenciados por el uso de estas redes.

Pero la existencia de estos espacios abre un nuevo campo para la investigación de los usuarios y del estudio de diferentes perfiles que pueden ser aprovechados en beneficio del emplazamiento de nuevos formatos publicitarios en la red.

Recordemos que en el año 2008 el incremento de la inversión publicitaria en Internet fue muy importante. Siendo este medio el que más ha crecido respecto al resto en el último año con un $28,2 \%$. Representando el $9 \%$ del total de la inversión publicitaria conocida (Zed Digital, 2008).

El dinamismo de los mercados y la exigencia de la demanda hacen que la competencia se establezca en parcelas muy concretas de dichos mercados, delimitadas por necesidades, deseos, intereses, preferencias y expectativas de consumidores que utilizan y se comunican a través de las nuevas posibilidades que ofrece Internet. Es por ello, que la aparición de las marcas con sus promociones y publicidad en estos nuevos espacios resulten de lo más rentable a las firmas comerciales, si además se tiene en cuenta que el público más influyente es el adolescente y más joven. Público objetivo que coincide como usuario de tuenti o Facebook. 


\section{METODOLOGÍA}

Con el fin de conocer el uso dado y la estructura de las redes sociales en el ámbito universitario, se seleccionó el universo de estudiantes de dos comunidades universitarias, la Universidad Europea Miguel de Cervantes, de titularidad privada y con sede en Valladolid, y la Universidad Francisco de Vitoria, también de titularidad privada y con sede en Madrid.

El método de obtención de información fue la encuesta estructurada. En dicha encuesta se utilizaron esencialmente preguntas dicotómicas, abiertas, cerradas y basadas en escalas. Se determinó el uso de escalas impares, de tipo acuerdo - desacuerdo en cinco posiciones, posibilitando la neutralidad en la opinión, ya que al ser un estudio a priori interesante para los encuestados se esperó una adecuada respuesta.

En cuanto a la técnica de muestreo, se utilizó el muestreo por cuotas. Se trataba de asegurar la adecuada representatividad del universo, por lo que se reprodujo en dicho muestreo la estructura del universo de estudio en razón de sexo y edad en primer nivel, y tomando en consideración en segundo lugar la licenciatura o diplomatura que estudian. El muestreo por cuotas constituye una síntesis de diferentes tipos y como intencionado, en todo caso ha de considerarse no probabilística, por lo que en principio no soporta la generalización de lo investigado. En definitiva, los resultados aquí presentados han de tomarse con la debida cautela.

El procedimiento de investigación se desarrolló durante el curso lectivo 2008-2009. El cuestionario contiene 15 preguntas - 260 variables - abiertas y cerradas, referentes a cuestiones de conocimiento en cuanto existencia, de frecuencia, de uso, estructura, preferencia y opinión.

Tabla 1. Cuestionario utilizado

\begin{tabular}{|l|l|}
\hline Ámbito & $\begin{array}{l}\text { Castilla y León / Valladolid / UEMC } \\
\text { Madrid / Pozuelo de Alarcón, (Madrid) / UFV }\end{array}$ \\
\hline Técnica & Cuestionario autoadministrado \\
\hline Población & 3455 Estudiantes \\
\hline Elementos de la muestra & Estratificada y cuotada. Muestra 264 sujetos \\
\hline
\end{tabular}

De la muestra seleccionada, la mayor parte coincide con la época teórica de estudio de licenciaturas y diplomaturas, es decir, de los 18 a los 24 años aproximadamente, guardando cierto equilibrio en razón de sexo, aunque con cierto predominio de las mujeres, siendo la misma compatible con el universo estudiado. 
Figura 1. Muestra seleccionada en razón de sexo y edad.

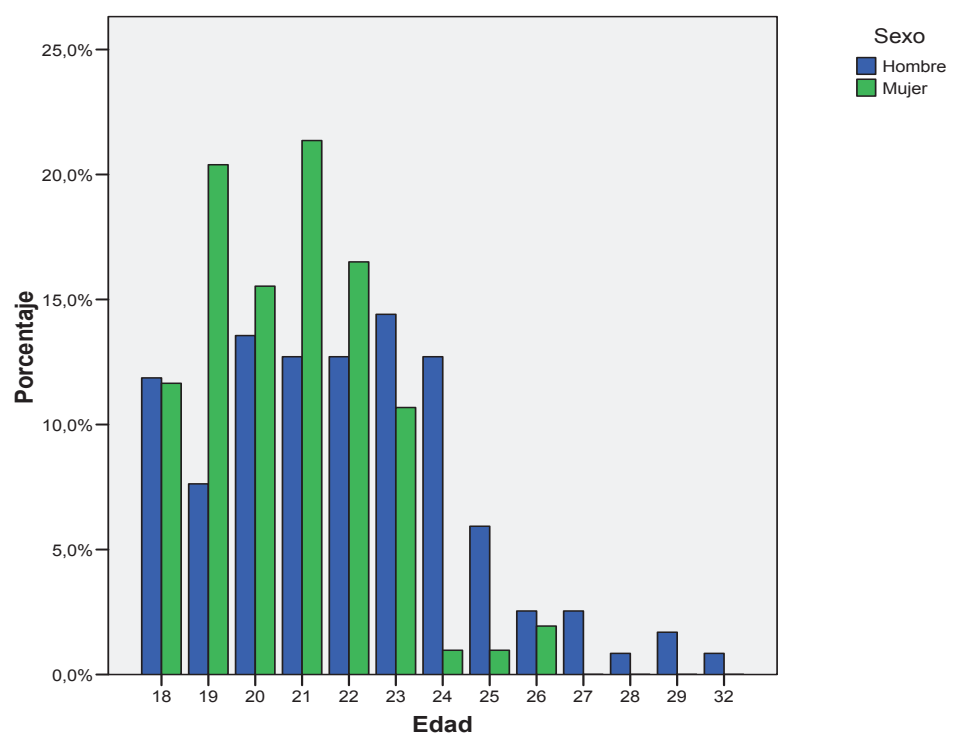

Fuente: Elaboración propia a partir de los resultados obtenidos

Para la explotación de datos se utilizó el software específico SPSS (Stadistical Package for Social Sciences) v. 16.

\section{RESULTADOS}

\subsection{Las redes sociales usadas}

En primer lugar, hay que señalar que el uso de redes sociales por la población objeto de estudio debe tildarse de masiva - el $86,7 \%$ de los hombres y el $93,2 \%$ de las mujeres afirman estar registrados en al menos una red social -. De los jóvenes que efectivamente estan registrados, la red social tuenti es la que ha conseguido acaparar la atención de los estudiantes univesitarios, ya que presenta un número de registros muy superior al resto de redes sociales. En menor medida pueden observarse cifras de registro relevantes para las redes Facebook, MySpace, hi5 y Fotolog. Finalmente, y considerando redes de escaso número de registros, situaríamos Ringo y Bebo. La categoría "otras" incluye principalmente a Messenger $(5,4 \%$ de usuarios registrados que la consideraron red social) - que aunque en puridad no debiera ser considerado como red social, o al menos la aplicación es estructuralmente muy disitnta a las tradicionales -, Badoo $(2,2 \%$ de registros) y Msn (2,7\% de registros efectivos). En menor medida se usan redes como Blogger, Coreo, Flickr, Fovos, NettBy, Radium y Studiva. 
Figura 2. Registros por red social (pregunta con respuesta múltiple).

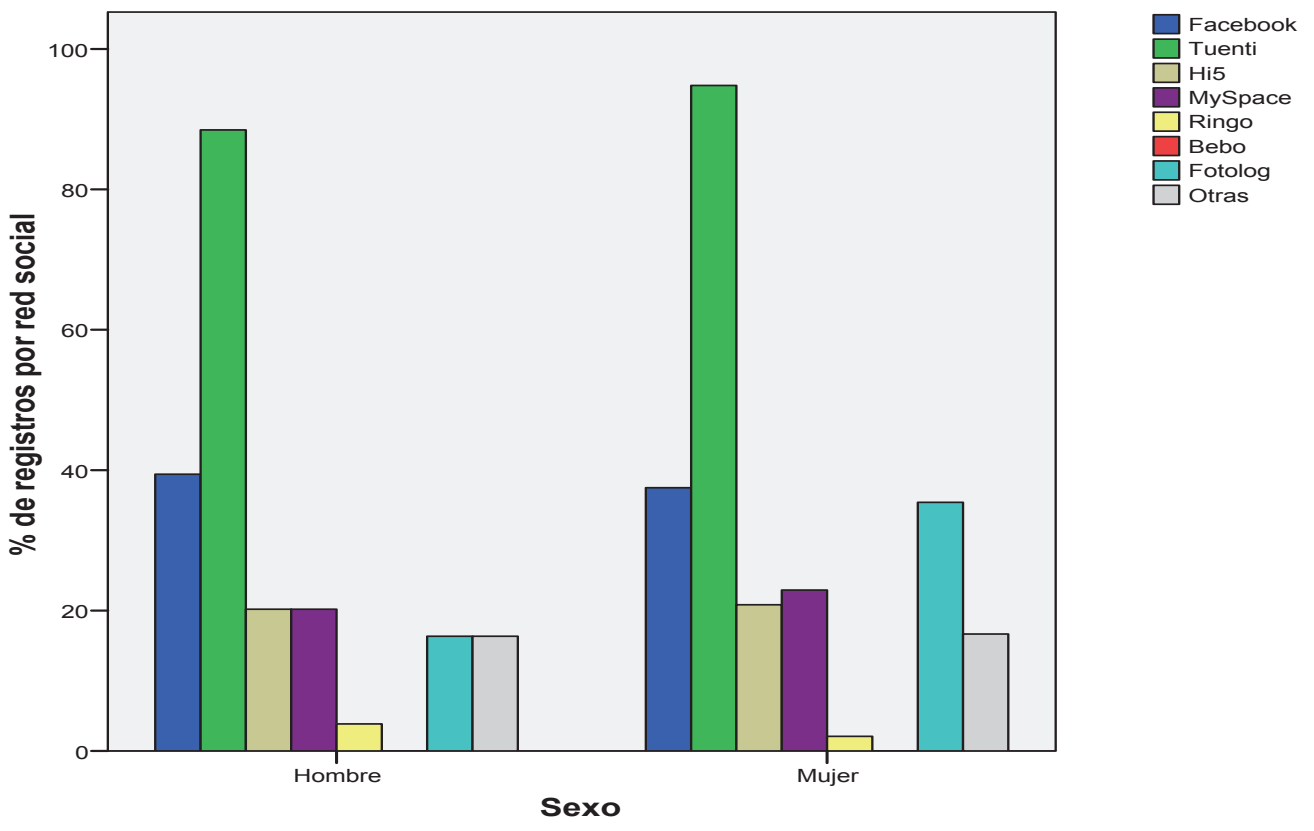

Fuente: Elaboración propia a partir de los resultados obtenidos

\subsection{Nomadismo virtual}

Sin embargo, el hecho de estar efectivamente registrado en una red social no implica necesariamente su uso habitual, pues es posible que en un momento del tiempo se decidiera el registro y posteriormente no se usara, o que se usara en el pasado y se hubiera dejado de hacerlo en el momento presente, bien debido a que en efecto se dejan de usar estas aplicaciones o bien porque se sustituye el uso de una por otra.

De hecho, una de las características fundamentales señaladas por la literatura de estas aplicaciones es que se encuadran en la tipología de "Killer Applications". Además funcionan en buena medida en base a modas, tienen un corto periodo de prevalencia entre sí y son sustituidas por los usuarios de unas a otras tras un breve periodo de permanencia en ambas, lo que permitiría señalar la existencia de un fenómeno de nomadismo virtual entre sus usuarios. Por ello, se pidió a los encuestados que revelaran para cada red si, en el caso de estar efectivamente registrado en la misma, se usaba de forma efectiva o no y para cada caso se estimara el número de meses.

Los resultados, compatibles con los aportados anteriormente, permiten sugerir alto grado de permanencia en tuenti, pues de los encuestados que se registraron en la misma el porcentaje de uso efectivo es del $97,8 \%$ de los hombres y el $98,9 \%$ de las mujeres. Menor grado se observa para Facebook $(76,2 \%$ de hombres y $77,8 \%$ de mujeres) y Fotolog (41,2\% de hombres y $55,9 \%$ de mujeres). Se observa cierto abandono en los casos de MySpace ( sólo el 38,1\% de los hombres registrados afirman utilizarla, y 
el 57,1\% de las mujeres), hi5 (19\% de los hombres y 20\% de las mujeres). Por último, Ringo (16,7\% para ambos sexos) y Bebo (sin registros efectivos).

No obstante, hay que considerar el tiempo que media entre el registro y su uso y abandono para poder sugerir la traslación de grupos de una aplicación a otra, circunstancia que confirmaría el carácter de Killer Applications de las redes sociales. Así, tuenti registra una relación muy estrecha y temporalmente reciente entre registro y uso efectivo (media aritmética en torno a los 11 meses en ambos casos), y como ya se ha dicho un grado de abandono muy bajo. Facebook y Fotolog muestran mayor indefinición, pues si bien la media aritmética se sitúa en torno a 6,3 meses en registro y uso o abandono para Facebook y 18 meses para Fotolog, la relación entre porcentaje antes señalado de uso efectivo y frecuencia temporal no permite concluir si Facebook es una aplicación en proceso de abandono o alza. Sin embargo, el caso de Fotolog es dintinto, pues el criterio temporal es en este caso muy superior, lo que permite sugerir que Fotolog tiene cierta base fiel de usuarios que se han mantenido durante largo tiempo. Estudios posteriores determinarán si se encuentra en proceso de abandono.

Más claros son los casos de hi5 (13 meses de registro con un alto porcentaje de abandono), MySpace (28 meses de registro por término medio y un alto nivel de abandono) y Ringo (17 meses de registro y altísimo nivel de abandono), para los que se puede sugerir que son aplicaciones en proceso de abandono.

También se les preguntó por su "red social favorita", pues cabía la posiblidad de que se registraran usos con intensidad parecida en varias redes sociales de forma simultánea, que funcionaran a modo de distintos grupos paralelos de amigos. Los resultados muestran una amplia compatibilidad con los datos aportados anteriormente, pues vuelve a ser tuenti la red social más respaldada (75\% de los hombres y $87,2 \%$ de las mujeres), seguida de Facebook (11,5\% de hombres y $7,4 \%$ de mujeres) y a gran distancia MySpace (opción señalada únicamente por el 2,1\% de hombres y mujeres), resultando la presencia del resto de redes inferior en este punto.

Por tanto, los datos señalados permiten sugerir que los grupos sociales virtuales estudiados podrían encontrarse en proceso de migración de MySpace, hi5 y Ringo hacia tuenti, y es posible que hacia Facebook. En consecuencia, cabe señalar un fenómeno de nomadismo virtual en estos usuarios de redes sociales, que "emigran" de una red social a otra sin permanecer mucho tiempo en cada una de ellas, sin establecer "residencia fija" en ninguna.

\subsection{Nomadismo virtual}

Otra cuestión interesante es sin duda conocer estructuralmente estas redes sociales virtuales, pues la estructura define en buena medida el modo de acceso a estos colectivos, especialmente en lo relativo a cómo se puede comunicar con mayor efectividad con estos grupos, o en qué modo focalizar acciones publicitarias o de 
relaciones públicas. Por ello recuperamos las ideas de Hunter (1953) y Dahl (1961) respecto de las teorías elitistas - concebidas fundamentalmente en relación a aspectos como clase social o reputación, con presencia muy marcada de la figura del líder - y pluralistas aplicadas a comunidades (Grunig y Hunt, 2003: 404-410) ${ }^{1}$, ya que entendemos que un grupo en red social virtual es esencialmente una comunidad virtual, un grupo de individuos con cierto interés común. También tenemos en cuenta el amplio estudio de Walton (1966) sobre centralización en comunidades.

En primer lugar debe considerarse que condición necesaria para que exista liderazgo en redes sociales es que éste debe ser explicitamente reconocido como tal por el resto, o al menos debe reconocerse que alguien orienta al resto ${ }^{2}$, no bastando por tanto que alguien simplemente use más algún aspecto de dicha red social para ser reconocido como líder. Con objeto de determinar estructura en las redes sociales, en esta investigación se preguntó, en una batería de 20 preguntas con escalas acuerdo desacuerdo de cinco posiciones que medían la existencia de líderes, la intensidad de uso de ciertos aspectos de las aplicaciones.

Se midió la consistencia interna de las escalas sobre la existencia de líderes y de personas orientadoras (con valor del estadístico Alfa de Cronbach en 0,512) y también de intensidad de uso de los diferentes aspectos de estas aplicaciones (con valor del mismo estadístico en 0,700). Adicionalmente, se examinó la coherencia de las respuestas mediante correlaciones de Spearman, que debían ser significativas e inversas en las preguntas de control (que se formularon en sentido opuesto, una respecto de la existencia de una figura de liderazgo y otra sobre la intensidad de uso). Los resultados señalan correlaciones estadísticamente significativas (ambas con grado de significación bilateral en 0.000 ) inversas con valores que oscilan entre -0.502 para el primer caso y 0.345 para el segundo.

Respecto de la autopercepción de intensidad de uso, los encuestados afirman mayoritariamente no ser ellos mismos quienes usan estas aplicaciones con mayor intensidad (59,5\% de los casos), si bien tampoco afirman ser los que menos las usan (45,8\% de los casos), y señalan usarlas con intensidad parecida al resto en general de integrantes de su red social (47,3\% de los casos), sin encontrarse diferencias relevantes en cuanto a sexo.

Sin embargo, si que consideran que "hay alguien" que en general usa más la red social que los demás (59,4\% de la muestra), tanto en general como en lo referente al envío de emails $(61,8 \%)$, subir y comentar fotografías $(74,4 \%)$, publicar y comentar noticias

1 Si bien las comunidades estudiadas eran norteamericanas, creemos que la esencia de dichas investigaciones es aplicable aquí.

2 En virtud de la definición de líder dada por la Real Academia de la Lengua. R.A.E Diccionario de la lengua española. Disponible en http://www.rae.es [Fecha de consulta 09/10/2010] 
(70,8\%), proponer el uso de aplicaciones (47,9\%) y escribir en los muros $(71,1 \%)$. Tampoco se obtuvieron diferencias relevantes respecto del sexo de los encuestados.

No obstante, y continuando con esta cuestión, mayoritariamente señalan que nadie orienta las conversaciones o la actividad de esa estructura $(78,8 \%)$, no reconociendo por tanto la figura de líder en estas redes virtuales. Ahondando un poco más en esta cuestión, se cuestionó si en esas estructuras existían subgrupos de usuarios que usaran con mayor intensidad que otros las redes sociales, y si los subgrupos que las usaban mucho o menos eran más o menos grandes en cuanto número de integrantes. Si bien mayoritariamente señalan la existencia de subgrupos con mayor intensidad de uso (50\% de los casos), los resultados no permiten señalar nada acerca del tamaño de los mismos.

También se consideró en otras dos preguntas que los grupos sociales virtuales formados tuvieran un referente real, es decir, que existieran grupos sociales virtuales que tuvieran referente físico (Alfa de Cronbach en 0,457). Respecto a esta cuestión, los encuestados señalan mayoritariamente y para las redes tuenti, Facebook, hi5, Fotolog y MySpace que conoció a los integrantes de la red social al margen de internet - con porcentajes que oscilan alrededor del $90 \%$ y sin encontrarse diferencias relevantes en cuanto a sexo -. Sin embargo, preguntados acerca de si mantienen el contacto con los mismos al margen de internet, en el caso de tuenti se muestra claramente - 99,4\% de encuestados, de los que el $9,9 \%$ es con todos, el 56,9\% con la mayoría y el 23,8\% es con aproximadamente la mitad de los integrantes -. El resto de redes sociales muestran una prevalencia superior en el caso de contacto entre miembros exclusivamente a través de las mismas - 8,5\% en el caso de Facebook, 27,3\% en el caso de hi5, 13,6\% en MySpace y 7,1\% en el caso de Fotolog -. En el resto de redes no hay datos suficientes para señalar tendencia alguna.

Así, se podría decir que -especialmente en el caso de tuenti - la comunicación en redes sociales virtuales tienen paralelismo en lo físico, pero no se puede afirmar que se reproduzcan estructuras físicas en ambientes virtuales, ya que si bien usualmente los usuarios se conocieron al margen de internet, los datos señalados en cuanto al mantenimiento de esa relación no permiten concluir una traslación de estructuras.

No obstante, si se puede afirmar que las redes sociales virtuales están formadas, al menos en parte, por estructuras físicas, ya sea una o varias. Es decir, no existe un paralelismo claro en que una estructura física se relacione con otra virtual, pero sí existe relación entre ambas, en algunos casos simétrica y en otros la segunda estará formada, al menos en parte, por una o varias estructuras físicas, y al mismo tiempo mantener componentes estrictamente virtuales. 


\section{CONCLUSIONES Y DISCUSIÓN DE RESULTADOS}

Los datos han permitido señalar en primer lugar un alto grado de registros en al menos una red social, lo que sugiere que nos encontramos ante un fenómeno de amplio espectro y consecuentemente de altas posibilidades de actuación en el campo comunicativo en general y publicitario y de relaciones públicas en particular. Actualmente, la red social tuenti parece ser la más popular entre los estudiantes universitarios. Sin embargo, hay que considerar el caracter nómada de sus usuarios, lo que exige especial vigilancia sobre las aplicaciones en las que realizar inversiones comunicativas.

La estructura de las redes sociales presenta cierta complejidad. Así, los encuestados no se autodefinen como intensos consumidores de estas redes, pero afirman la existencia de subgrupos con consumos de estas aplicaciones más y menos intensos dentro de la estructura principal, en número variable de integrantes, y de perfiles especialmente activos, pero no los reconocen como líderes u orientadores de conversación o actividad. Por tanto, no podemos afirmar la existencia de una estructura elitista, pero sí de una estructura pseudopluralista con algunos rasgos propios de estructuras elitistas, al menos en cuanto a que se reconoce la existencia de subgrupos y también de perfiles individuales especialmente activos -sin la condición de líderes- que pudieran resultar interesantes para la focalización de acciones comunicativas y que podrían ser eficientes transmisores de información.

En cuanto a la posible traslación de estructuras con referente físico a estructuras estrictamente virtuales, no podemos concluirla. Si bien los integrantes de la redes sociales afirman conocer a la mayoría del resto al margen de esta tecnología, no puede afirmarse que se produzca simetría en la traslación, pues también mayoritariamente afirman que en las redes sociales existen integrantes conocidos dentro de la red, y además el hecho de conocerse fuera de internet no implica que se pertenezca a la misma estructura física, pudiendo por tanto existir comunidades virtuales en redes sociales pertenecientes a distintas estructuras físicas, o resultando ser las primeras integradoras de varias físicas y una o varias estrictamente virtuales. Por tanto, una estrategia comunicativa - o de relaciones públicas - adecuada deberá contemplar y asumir la falta de simetría en ambos contextos, físico y virtual, y por tanto considerar acciones exclusivamente ideadas y focalizadas en ambos contextos, lo que tiene notables implicaciones en el mensaje a transmitir o la acción a realizar.

Las limitaciones más importantes de este trabajo se refieren a la muestra utilizada, tanto por el número de elementos muestrales usados como en las propias caracerísticas de los mismos, ya que únicamente se han considerado alumnos de universidad privada, que tienen un perfil específico que pudiera diferir notablemente de los alumnos de universidad pública. 
Finalmente, futuras líneas de investigación podrían contemplar la profundización del estudio incorporando nuevas variables en la clasificación de estructuras, tales como la toma de decisiones o la conflictividad en estos sistemas virtuales. También podría resultar de interés la observación de dichos las redes sociales en relación a la aportación de estos grupos virtuales en la autopercepción de los individuos dentro y fuera de estas estructuras.

\section{BIBLIOGRAFÍA}

- DAHL, R. (1961) Who governs ? Yale University Press.

- ELOLA, J. (2008) “O estás en tuenti o perdiste el tren” Diario El País.

Disponible en http://www.elpais.com/articulo/sociedad/Tuenti/perdiste/tren/elpepisoc /20081214elpepisoc_3/Tes

- EROSKI CONSUMER (2010) "Un estudio sitúa a los españoles entre los europeos más activos en redes sociales". Disponible en http://www.consumer.es/

- FERNÁNDEZ, S. (2008) “Redes Sociales. Fenómeno pasajero o reflejo del nuevo internauta". Telos, 76, 118.

- FLOREZ, J. L.(2008) "Análisis de redes sociales: el poder de la influencia" Harvard - Deusto Marketing \& Ventas, 86, 62-70.

- FUMERO, A HERVÁS y GARCÍA, JM, (2008). “Redes Sociales.

Contextualización de un fenómeno "dos-punto-cero" $\left({ }^{*}\right)$ " Revista Telos, 76.

- GRUNIG, J.E. y HUNT, T. Dirección de Relaciones Públicas. Ed Gestión 2000, Barcelona, 2003. ed. XIFRÁ, J. 
- HUNTER, F.(1953) Community power structure: a study of decision makers University of North Carolina Press.

- IAB (2009) “Estudio sobre redes sociales en España 2009”. Disponible en http://www.iabspain.biz

- KATZ, R..L., CHROUSOS, P. y WU, H.(2008) “La sobrevaloración de las redes sociales en internet". Enter-IE, 81, 5.

- ORIHUELA, J. L (2008). “Internet: la hora de las redes sociales”. Nueva Revista, $119,57-62$.

- ROCA, G. (2008) Soluciones para nuevas realidades. Modelos de negocio en las redes sociales. Telos, 76 .

- SÁNCHEZ REVILLA, M.A., La publicidad bien, Memoria Infoadex 2008, Madrid, 2008. Disponible en: http:/ / www.agemdi.org

- THE COCKTAIL ANALYSIS (2008) “Herramientas de comunicación on-line: Las Redes Sociales." Disponible en http:/ / www.tcanalysis.com

- WALTON, J. (1966) "Discipline, method and community power: A note on the sociology of knowledge". American Sociologial Review, 31,

- ZED DIGITAL (2008) "Primer estudio sobre redes sociales y publicidad" Disponible en: http:/ /www.data-red.com/

- ZED DIGITAL (2008) “Estudio sobre el Fenómeno de las Redes Sociales en España: percepción, usos y publicidad". Disponible en http:/ / www.zeddigital.es/ 